\title{
DISTRIBUTIONS IN LIFE INSURANCE
}

\author{
By Jan DhaEne \\ Instituut voor Actuariële Wetenschappen, \\ K.U. Leuven, Belgium
}

\begin{abstract}
In most textbooks and papers that deal with the stochastic theory of life contingencies, the stochastic approach is restricted to the computation of expectations and higher order moments. For a wide class of insurances on a single life, we derive the distribution and the probability density function of the benefit and the loss functions. Both the continuous and the discrete case are considered.
\end{abstract}

\section{KEYWORDS}

Single life contingencies; benefit function; loss function; stochastic approach.

\section{INTRODUCTION}

In the two recent actuarial textbooks of GERBER (1986) and Bowers et al. (1987) the theory of life contingencies is built up in function of the stochastic remaining life time of the insured.

This stochastic approach permits to define two important kinds of stochastic functions for an insurance: the benefit function and the loss function at a certain time. The benefit function of an insured of age $x$ at policy issue is defined as the discounted value of all the benefits to be paid by the insurer over the random future lifetime $T_{x}$ of the insured. The loss function at time $s$, given the insured is alive at that time, is the discounted value of all the benefits to be paid by the insurer over the random future lifetime $T_{x+s}$ of the insured less the discounted value of all the premiums to be paid by the insured over the same period.

Most results of the traditional deterministic theory are obtained by considering only the expected value of the above defined functions. The net single premium is defined as the expectation of the benefit function. The equivalence principle is the requirement that the expected loss at time 0 equals 0 . From this requirement the net premiums can be computed. The net premium reserve at time $s$ is defined as the expectation of the loss function at time $s$.

Bowers et al. (1987) state that the probabilistic approach of life contingencies "admits a rich field of random variable concepts such as distribution 
function, probability density function, expected value, variance and moment generating function". Nevertheless, the literature on this probabilistic approach is mostly restricted to the computation of moments of the benefit and the loss functions, see e.g. Pollard and Pollard (1969), Wolthuis and Van Hoek (1984), Gerber (1986) and Bowers et al. (1987).

DE PRIL (1989) gives a survey of the distribution functions (d.f.) and the probability density functions (p.d.f.) of the benefit function of most common life insurances and annuities.

In this paper we will consider the benefit and loss functions of a "general insurance", by which we mean a combination of the commonly used life insurances, endowment insurances and life annuities. It will be shown that these functions are random variables of a special type. The d.f. and the p.d.f. of a random variable of this type will then be derived. For completeness both the continuous and discrete case will be treated.

\section{CONTINUOUS DESCRIPTION OF SINGLE LIFE CONTINGENCIES}

Let $T_{x} \equiv T$ be a continuous nonnegative random variable representing the future lifetime of a life-aged- $x$.

Using the common actuarial notation, the d.f. of $T$ can be written as

$$
F_{T}(t)=\operatorname{Prob}(T \leq t)= \begin{cases}0 & t<0 \\ { }_{t} q_{x}=1-{ }_{t} p_{x} & t \geq 0\end{cases}
$$

TABLE 1

CONSTANTS FOR THE CONTINUOUS ACTUARIAL FUNCTIONS

L.I. = Life Insurance, E.I. = Endowment Insurance, L.A. = Life Annuity

\begin{tabular}{lcccccc}
\hline \hline Name & Notation & $a$ & $b$ & $c$ & $m$ & $n$ \\
\hline whole L.I. & $\bar{A}_{x}$ & 0 & 1 & 0 & 0 & $\infty$ \\
$n$-year term L.I. & $\bar{A}_{x n\urcorner}^{\prime}$ & 0 & 1 & 0 & 0 & $n$ \\
$m$-year deferred L.I. & ${ }_{m \mid} \bar{A}_{x}$ & 0 & 1 & 0 & $m$ & $\infty$ \\
$m$-year deferred & ${ }_{m \mid} \bar{A}_{x n\urcorner}^{\prime}$ & 0 & 1 & 0 & $m$ & $n$ \\
$n$-year term L.I. & $\left.\left.\bar{A}_{x} 1\right\urcorner\right\urcorner$ & 0 & 0 & $v^{n}$ & 0 & $n$ \\
$n$-year pure E.I. & $\bar{A}_{x n\urcorner}$ & 0 & 1 & $v^{n}$ & 0 & $n$ \\
$n$-year E.I. & $m \mid \bar{A}_{x n\urcorner}$ & 0 & 1 & $v^{m+n}$ & $m$ & $n$ \\
$m$-year deferred & $\bar{a}_{x}$ & $1 / \delta$ & $-1 / \delta$ & 0 & 0 & $\infty$ \\
$n$-year E.I. & $\bar{a}_{x n\urcorner}$ & $1 / \delta$ & $-1 / \delta$ & $\bar{a}_{n\urcorner}$ & 0 & $n$ \\
whole L.A. & ${ }^{\prime} \bar{a}_{x}$ & $v^{m} / \delta$ & $-1 / \delta$ & 0 & $m$ & $\infty$ \\
$n$-year temporary L.A. & ${ }_{m \mid} \bar{a}_{x n\urcorner}$ & $v^{m} / \delta$ & $-1 / \delta$ & $v^{m} \bar{a}_{n\urcorner}$ & $m$ & $n$ \\
$m$-year deferred whole L.A. & & & & & \\
$m$-year deferred & & & & &
\end{tabular}


with ${ }_{0} q_{x}=0$ and $\lim _{t \rightarrow \infty}, q_{x}=1$.

The p.d.f. of $T$ is given by

$$
f_{T}(t)=F_{T}^{\prime}(t)= \begin{cases}0 & t<0 \\ { }_{t} p_{x} \quad \mu_{x+t} & t \geq 0\end{cases}
$$

where $\mu_{x}$ denotes the force of mortality of a life aged $x$.

From Table 1 it can be seen that the benefit function of the common life insurances, endowment insurances and life annuities on a single life aged $x$ at policy issue can be written as a stochastic variable of the form

$$
S= \begin{cases}0 & 0 \leq T<m \\ a+b v^{T} & m \leq T<m+n \\ c & T \geq m+n\end{cases}
$$

where $a, b$ and $c$ are real numbers and $m$ and $n$ are nonnegative integers. Further, $v=1 /(1+i)$ is the present value factor related to the annual valuation rate of interest $i$.

In Table 1 the following notation is used: $\delta=\ln (1+i)$ is the force of interest associated with the valuation rate of interest $i$ and $\bar{a}_{n\urcorner}=\left(1-v^{n}\right) / \delta$ is a continuous $n$-year temporary annuity certain.

A general continuous insurance on a single life aged $x$ at policy issue is defined as a combination of the life insurances, endowment insurances and life annuities considered in Table 1 and where the premiums are paid by a combination of the life annuities and pure endowment insurances of Table 1.

The stochastic variable describing the benefit function of a general continuous insurance is a linear combination of random variables of the form (2.3). So it follows immediately that this stochastic variable can be written as

$$
S=a_{i}+b_{i} v^{T} \quad m(i-1) \leq T<m(i) ; \quad i=1, \ldots, n
$$

with $T \equiv T_{x}, a_{i}$ and $b_{i}(i=1, \ldots, n)$ real numbers and $m(i)(i=0, \ldots, n)$ nonnegative integers satisfying

$$
0 \leq m(0)<m(1)<\ldots<m(n) \leq \infty
$$

It is easy to see that the loss function at times $s(s \geq 0)$, given survival of the insured at that time, can also be described by a stochastic variable of the form (2.4) with $T \equiv T_{x+s}$.

The p.d.f. of a random variable of the form (2.4) will be derived in the following theorem. The delta-function will be denoted by $\Delta(x)$ to avoid confusion with the symbol $\delta$ for the force of interest. For a study of the delta-function see e.g. PAPOULIS (1962). 
Theorem 1. Let $S$ be the stochastic variable defined in (2.4) with $T \equiv T_{z}$.

Define for $i=1, \ldots, n$

$$
\begin{aligned}
& m(i)^{-}=a_{i}+\min \left(b_{i} v^{m(i-1)}, b_{i} v^{m(i)}\right) \\
& m(i)^{+}=a_{i}+\max \left(b_{i} v^{m(i-1)}, b_{i} v^{m(i)}\right)
\end{aligned}
$$

The p.d.f. of $S$ is given by

$$
f(s)=\sum_{i=1}^{n} G_{i}(s)
$$

with for $i=1, \ldots, n$

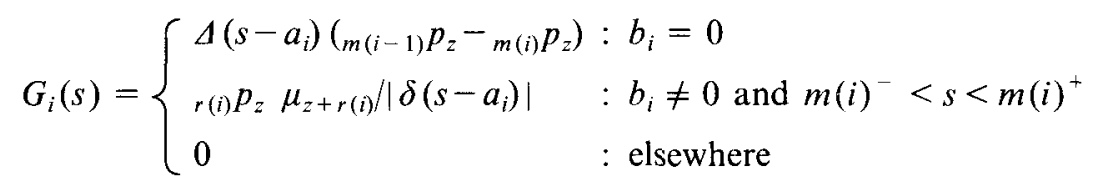

where $r(i)$ is given by

$$
r(i)=-\frac{1}{\delta} \ln \left(\frac{s-a_{i}}{b_{i}}\right) \quad \frac{s-a_{i}}{b_{i}}>0
$$

Proof. Using the Law of Total Probability the p.d.f. of $S$ can be written in the form (2.8) with

$$
G_{i}(s)=f(s \mid m(i-1) \leq T<m(i)) \operatorname{Prob}(m(i-1) \leq T<m(i))
$$

For $b_{i}=0$ it follows that

$$
f(s \mid m(i-1) \leq T<m(i))=\Delta\left(s-a_{i}\right) .
$$

Consider now the case $b_{i} \neq 0$. We obtain

$G_{i}(s)= \begin{cases}f_{T}(r(i)) /\left(\delta\left(s-a_{i}\right)\right) & : b_{i}>0, a_{i}+b_{i} v^{m(i)}<s<a_{i}+b_{i} v^{m(i-1)} \\ f_{T}(r(i)) /\left(\delta\left(a_{i}-s\right)\right) & : b_{i}<0, a_{i}+b_{i} v^{m(i-1)}<s<a_{i}+b_{i} v^{m(i)} \\ 0 & : \text { elsewhere. }\end{cases}$

with $r(i)$ defined in (2.10).

So it follows that $G_{i}(s)$ is given by (2.9).

The d.f. of $S$ is derived in the next theorem. The following notation will be used :

$$
(x)_{+}=\max (0, x)
$$

and

$$
H(x)=\left\{\begin{array}{l}
0: x<0 \\
1: x \geq 0
\end{array}\right.
$$


Theorem 2. The d.f. of the random variable $S$ defined in (2.4) with $T \equiv T_{z}$ is given by

$$
F(s)=\sum_{i=1}^{n}\left({ }_{a(i)} q_{z}-{ }_{\beta(i)} q_{z}\right)_{+} K_{i}(s)
$$

where $a(i)$ and $\beta(i)(i=1, \ldots, n)$ are given by

(2.13) $a(i)= \begin{cases}\max \left\{0, \min \left\{m(i),-\frac{1}{\delta} \ln \left(\frac{s-a_{i}}{b_{i}}\right)\right\}\right\} & : b_{i}<0, s<a_{i} \\ m(i) & : \text { elsewhere }\end{cases}$
(2.14) $\beta(i)= \begin{cases}m(i-1) & : b_{i} \leq 0 \\ \max \left\{m(i-1),-\frac{1}{\delta} \ln \left(\frac{s-a_{i}}{b_{i}}\right)\right\} & : b_{i}>0, s>a_{i} \\ m(i) & : b_{i}>0, s \leq a_{i}\end{cases}$

Finally, $K_{i}(s)(i=1, \ldots, n)$ is defined as

$$
K_{i}(s)= \begin{cases}H\left(s-a_{i}\right) & : b_{i}=0 \\ 1 & : b_{i} \neq 0\end{cases}
$$

Proof. Using the Law of Total Probability we find that

$$
F(s)=\sum_{i=1}^{n} \operatorname{Prob}\left(a_{i}+b_{i} v^{T} \leq s \text { and } m(i-1) \leq T<m(i)\right)
$$

It follows that

$\operatorname{Prob}\left(a_{i}+b_{i} v^{T} \leq s\right.$ and $\left.m(i-1) \leq T<m(i)\right)$

$$
= \begin{cases}H\left(s-a_{i}\right) \operatorname{Prob}(m(i-1) \leq T<m(i)) & : b_{i}=0 \\ \operatorname{Prob}\left(\max \left\{m(i-1),-\frac{1}{\delta} \ln \left(\frac{s-a_{i}}{b_{i}}\right)\right\} \leq T<m(i)\right) & : b_{i}>0, s>a_{i} \\ 0 & : b_{i}>0, s \leq a_{i} \\ \operatorname{Prob}(m(i-1) \leq T<m(i)) & : b_{i}<0, s \geq a_{i} \\ \operatorname{Prob}\left(m(i-1) \leq T<\min \left\{m(i),-\frac{1}{\delta} \ln \left(\frac{s-a_{i}}{b_{i}}\right)\right\}\right) & : b_{i}<0, s<a_{i}\end{cases}
$$

Or

$$
\operatorname{Prob}\left(a_{i}+b_{i} v^{T} \leq s \text { and } m(i-1) \leq T<m(i)\right)=\left({ }_{a(i)} q_{z}-\beta(i) q_{z}\right)+K_{i}(s)
$$


with $a(i)$ and $\beta(i)$ defined in (2.13) and (2.14).

Now (2.12) is obtained with the help of (2.16).

The p.d.f. and the d.f. of the benefit function of the insurances and annuities considered in Table 1 can be written in a simpler form as is proven in the following corollary.

Corollary 1. Let $S$ be the stochastic variable defined in (2.3) with $T \equiv T_{x}$.

Define

$$
\begin{aligned}
& m^{-}=a+\min \left(b v^{m}, b v^{m+n}\right) \\
& m^{+}=a+\max \left(b v^{m}, b v^{m+n}\right)
\end{aligned}
$$

The p.d.f. of $S$ is given by

$$
f(s)={ }_{m} q_{x} \Delta(s)+G(s)+{ }_{m+n} p_{x} \Delta(s-c)
$$

where $G(s)$ is defined as

$$
G(s)= \begin{cases}\Delta(s-a)\left({ }_{m} p_{x}-{ }_{m+x} p_{x}\right) & : b=0 \\ { }_{r} p_{x} \mu_{x+r} /|\delta(s-a)| & : b \neq 0 \text { and } m^{-}<s<m^{+} \\ 0 & : \text { elsewhere }\end{cases}
$$

with

$$
r=-\frac{1}{\delta} \ln \left(\frac{s-a}{b}\right) \quad \frac{s-a}{b}>0
$$

The d.f. of $S$ is given by

$$
F(s)={ }_{m} q_{x} H(s)+\left({ }_{a} q_{x}-{ }_{\beta} q_{x}\right)+K(s)+{ }_{m+n} p_{x} H(s-c)
$$

with

$$
a= \begin{cases}\max \left\{0, \min \left\{m+n,-\frac{1}{\delta} \ln \left(\frac{s-a}{b}\right)\right\}\right\} & : b<0, s<a \\ m+n & : \text { elsewhere }\end{cases}
$$

(2.24) $\beta= \begin{cases}m & : b \leq 0 \\ \max \left\{m,-\frac{1}{\delta} \ln \left(\frac{s-a}{b}\right)\right\} & : b>0, s>a \\ m+n & : b>0, s \leq a\end{cases}$ 

(2.25) $K(s)=\left\{\begin{array}{l}H(s-a) \\ 1\end{array}\right.$
$: b=0$
$: b \neq 0$

Proof. The random variable $S$ defined in formula (2.3) is a special case of the random variable defined by (2.4) with the constants $n=3, a_{1}=0, b_{1}=0$, $a_{2}=a, \quad b_{2}=b, \quad a_{3}=c, \quad b_{3}=0, \quad m(0)=0, \quad m(1)=m, \quad m(2)=m+n$, $m(3)=\infty$ and $T \equiv T_{x}$.

Using Theorems 1 and 2, after some straightforward calculation one obtains formulae (2.19) and (2.22).

The d.f. and the p.d.f. of all the continuous insurances and annuities considered in De PrIL (1989) can be obtained by using Table 1 and Corollary 1.

TABLE 2

CONSTANTS FOR THE DISCRETE ACTUARIAL FUNCTIONS

L.I. = Life Insurance, E.I. = Endowment Insurance, L.A. $=$ Life Annuity

\begin{tabular}{|c|c|c|c|c|c|c|}
\hline Name & Notation & $a$ & $b$ & $c$ & $m$ & $n$ \\
\hline whole L.I. & $A_{x}$ & 0 & 1 & 0 & 0 & $\infty$ \\
\hline$n$-year term L.I. & $\left.A_{x}^{1} \cap\right\urcorner$ & 0 & 1 & 0 & 0 & $n$ \\
\hline m-year deferred L.I. & ${ }_{m \mid} \boldsymbol{A}_{x}$ & 0 & 1 & 0 & $m$ & $\infty$ \\
\hline $\begin{array}{l}m \text {-year deferred } \\
n \text {-year term L.l. }\end{array}$ & $m \mid \bar{A}_{x n\urcorner}^{1}$ & 0 & 1 & 0 & $m$ & $n$ \\
\hline n-year pure E.I. & $A_{x} \stackrel{1}{7}$ & 0 & 0 & $v^{n}$ & 0 & $n$ \\
\hline n-year E.I. & $\left.A_{x n\urcorner}\right\urcorner$ & 0 & 1 & $v^{n}$ & 0 & $n$ \\
\hline $\begin{array}{l}m \text {-year deferred } \\
n \text {-year E.I. }\end{array}$ & ${ }_{m \mid} \boldsymbol{A}_{x n\urcorner}$ & 0 & 1 & $v^{m+n}$ & $m$ & $n$ \\
\hline whole L.A. due & $\ddot{a}_{x}$ & $1 / d$ & $-1 / d$ & 0 & 0 & $\infty$ \\
\hline whole L.A. immediate & $a_{x}$ & $1 / i$ & $-1 / d$ & 0 & 0 & $\infty$ \\
\hline$n$-year temporary L.A. due & $\ddot{a}_{x n\urcorner}$ & $1 / d$ & $-1 / d$ & $\ddot{a}_{n\urcorner}$ & 0 & $n$ \\
\hline $\begin{array}{l}n \text {-year temporary L.A. } \\
\text { immediate }\end{array}$ & $a_{x n\urcorner}$ & $1 / i$ & $-1 / d$ & $a_{n\urcorner}$ & 0 & $n$ \\
\hline $\begin{array}{l}m \text {-year deferred whole L.A. } \\
\text { due }\end{array}$ & $m \mid \ddot{a}_{x}$ & $v^{m} / d$ & $-1 / d$ & 0 & $m$ & $\infty$ \\
\hline $\begin{array}{l}m \text {-year deferred } \\
\text { whole L.A. immediate }\end{array}$ & $m \mid a_{x}$ & $v^{m} / i$ & $-1 / d$ & 0 & $m$ & $\infty$ \\
\hline $\begin{array}{l}m \text {-year deferred } \\
n \text {-year temporary L.A. due }\end{array}$ & $m \mid \ddot{a}_{x n\urcorner}$ & $v^{m} / d$ & $-1 / d$ & $v^{m} \ddot{a}_{n\urcorner}$ & $m$ & $n$ \\
\hline $\begin{array}{l}m \text {-year deferred } \\
n \text {-year temporary L.A. } \\
\text { immediate }\end{array}$ & $m \mid a_{x n\urcorner}$ & $v^{m} / i$ & $-1 / d$ & $v^{m} \ddot{a}_{n\urcorner}$ & $m$ & $n$ \\
\hline
\end{tabular}




\section{DISCRETE DESCRIPTION OF SINGLE LIFE CONTINGENCIES}

Let $K \equiv K_{x}$ be a nonnegative random variable, representing the number of full years to death of a life-aged- $x$.

The distribution of $K$ can then be written as

$$
F_{K}(k)=\operatorname{Prob}(K \leq k)={ }_{k+1} q_{x}=1-{ }_{k+1} p_{x} \quad k=0,1,2, \ldots
$$

with $\lim _{k \rightarrow \infty}{ }_{k} q_{x}=1$

The p.d.f. of $K$ is given by

$$
f_{K}(k)={ }_{k} p_{x}-{ }_{k+1} p_{x}={ }_{k} q_{x} \quad k=0,1,2, \ldots
$$

The benefit functions of the common discrete life insurances, endowment insurances and life annuities on a single life aged $x$ at policy issue can be defined as stochastic variables of the form

$$
S= \begin{cases}0 & : K=0,1, \ldots, m-1 \\ a+b v^{K+1} & : K=m, m+1, \ldots, m+n-1 \\ c & : K=m+n, m+n+1, \ldots\end{cases}
$$

The suiting values for $a, b, c, m$ and $n$ are given in Table 2 .

The following notation is used: $\left.d=1-v, \ddot{a}_{n}\right\urcorner=\left(1-v^{n}\right) / d$ is a discrete $n$-year temporary annuity due and $a_{n\urcorner}=\left(1-v^{n}\right) / i$ is a discrete $n$-year temporary annuity immediate.

A general discrete insurance on a single life-aged- $x$ is defined as a combination of the insurances defined in Table 2. The premiums are paid by a combination of the life annuities and pure endowment insurances of Table 2.

The benefit function and the loss function of a general discrete insurance can be described by a stochastic variable $S$ of the form

$$
S=a_{i}+b_{i} v^{K+1} \quad m(i-1) \leq K<m(i) ; \quad i=1, \ldots, n
$$

with $K \equiv K_{x}$ for the benefit function and $K \equiv K_{x+s}$ for the loss function at time s. Further, $a_{i}$ and $b_{i}(i=1, \ldots, n)$ are real numbers and $m(i)$ $(i=0,1, \ldots, n)$ are nonnegative integers satisfying

$$
0 \leq m(0)<m(1)<\ldots<m(n) \leq \infty
$$

In the following theorems the p.d.f. and the d.f. of $S$ are derived.

Theorem 3. The p.d.f. of the variable $S$ defined in (3.4) where $K \equiv K_{z}$ is given by

$$
f(s)=\sum_{i=1}^{n} G_{i}(s)
$$


where for $i=1, \ldots, n$ the functions $G_{i}(s)$ are given by

$$
G_{i}(s)= \begin{cases}\left.{ }_{m(i-1)} p_{z}-{ }_{m(i)} p_{z}\right) \Delta\left(s-a_{i}\right) & : b_{i}=0 \\ \sum_{k=m(i-1)}^{m(i)-1} k \mid q_{z} \Delta\left(s-a_{i}-b_{i} v^{k+1}\right) & : b_{i} \neq 0\end{cases}
$$

Proof. For $m(i-1) \leq K<m(i)$ and $b_{i}=0$ we get that $S=a_{i}$ or

$$
f(s \mid m(i-1) \leq K<m(i))=\Delta\left(s-a_{i}\right)
$$

If $m(i-1) \leq K<m(i)$ and $b_{i} \neq 0$ the possible values for $S$ are

$$
a_{i}+b_{i} v^{k+1} \quad k=m(i-1), \ldots, m(i)-1
$$

with respective probabilities

$$
{ }_{k \mid} q_{x} / \operatorname{Prob}(m(i-1) \leq K<m(i))
$$

So we find for $b_{i} \neq 0$

$$
\begin{aligned}
& f(s \mid m(i-1) \leq K<m(i)) \operatorname{Prob}(m(i-1) \leq K<m(i)) \\
& =\sum_{k=m(i-1)}^{m(i)-1} k_{k} q_{x} \Delta\left(s-a_{i}-b_{i} v^{k+1}\right)
\end{aligned}
$$

By using the Law of Total Probability we obtain formula (3.6).

Theorem 4. The d.f. of the random variable $S$ defined in (3.4) where $K \equiv K_{z}$ is given by

$$
F(s)=\sum_{i=1}^{n}\left({ }_{a(i)} q_{z}-\beta(i) q_{z}\right)_{+} K_{i}(s)
$$

where for $i=1, \ldots, n$ the functioins $a(i), \beta(i)$ and $K_{i}(s)$ are given by

$$
\begin{aligned}
\text { (3.9) } a(i)= \begin{cases}\max \left\{0, \min \left\{m(i),\left[-\frac{1}{\delta} \ln \left(\frac{s-a_{i}}{b_{i}}\right)\right]\right\}\right\} & : b_{i}<0, s<a_{i} \\
m(i) & : \text { elsewhere }\end{cases} \\
\text { (3.10) } \beta(i)= \begin{cases}m(i-1) & : b_{i} \leq 0 \\
\max \{m(i-1),]-\frac{1}{\delta} \ln \left(\frac{s-a_{i}}{b_{i}}\right)-1[\} & : b_{i} \leq 0, s>a_{i} \\
m(i) & : b_{i}>0, s \leq a_{i}\end{cases}
\end{aligned}
$$


(3.11) $\quad K_{i}(s)=\left\{\begin{array}{l}H\left(s-a_{i}\right) \\ 1\end{array}\right.$

$$
\begin{aligned}
& : b_{i}=0 \\
& : b_{i} \neq 0
\end{aligned}
$$

For a real number $x]$,$x [ denotes the smallest integer greater than or equal to x$ and $[x]$ denotes the greatest integer less than or equal to $x$.

Proof. For $\mathrm{m}(i-1) \leq K<m(i)$ we find

$\operatorname{Prob}\left(a_{i}+b_{i} v^{K+1} \leq s\right.$ and $\left.m(i-1) \leq K<m(i)\right)$

$$
= \begin{cases}H\left(s-a_{i}\right) \operatorname{Prob}(m(i-1) \leq K<m(i)) & : b_{i}=0 \\ \operatorname{Prob}\left(\max \{m(i-1),]-\frac{1}{\delta} \ln \left(\frac{s-a_{i}}{b_{i}}\right)-1[\} \leq K<m(i)\right) & : b_{i}>0, s>a_{i} \\ 0 & : b_{i}>0, s \leq a_{i} \\ \operatorname{Prob}(m(i-1) \leq K<m(i)) & : b_{i}<0, s \geq a_{i} \\ \operatorname{Prob}\left(m(i-1) \leq K<\min \left\{m(i),\left[-\frac{1}{\delta} \ln \left(\frac{s-a_{i}}{b_{i}}\right)\right]\right\}\right) & : b_{i}<0, s<a_{i}\end{cases}
$$

Or

Prob $\left(a_{i}+b_{i} v^{K+1} \leq s\right.$ and $\left.m(i-1) \leq K<m(i)\right)=\left({ }_{a(i)} q_{z}-{ }_{\beta(i)} q_{z}\right)_{+} K_{i}(s)$ with $a(i), \beta(i)$ and $K_{i}(s)$ defined in (3.9), (3.10) and (3.11).

By using the Law of Total Probalility we obtain the desired result.

The p.d.f. and the d.f. of the benefit function of the discrete insurances and annuities considered in Table 2 can be written in a simpler form which is derived in the next corollary.

Corollary 2. Let $S$ be the stochastic variable defined in (3.3) with $K \equiv K_{x}$. The p.d.f. of $S$ is given by

$$
f(s)={ }_{m} q_{x} A(s)+G(s)+{ }_{m+n} p_{x} \Delta(s-c)
$$

with

$$
G(s)= \begin{cases}\left.{ }_{m} p_{x}-{ }_{m+n} p_{x}\right) \Delta(s-a) & : b=0 \\ \sum_{k=m}^{m+n-1}{ }_{k \mid} q_{x} \Delta\left(s-a-b v^{k+1}\right) & : b \neq 0\end{cases}
$$

The d.f. of $S$ is given by

$$
F(s)={ }_{m} q_{x} H(s)+\left({ }_{a} q_{x}-{ }_{\beta} q_{x}\right)+K(s)+{ }_{m+n} p_{x} H(s-c)
$$


with

$$
\begin{aligned}
& \text { (3.15) } a= \begin{cases}\max \left\{0, \min \left\{m+n,\left[-\frac{1}{\delta} \ln \left(\frac{s-a}{b}\right)\right]\right\}\right\} & : b<0, s<a \\
m+n & : \text { elsewhere }\end{cases} \\
& \text { (3.16) } \quad \beta= \begin{cases}m & : b \leq 0 \\
\max \{m, & : b>0, s>a \\
m+n & : 1-\frac{1}{\delta} \ln \left(\frac{s-a}{b}\right)[\}\end{cases} \\
& \text { (3.17) } K(s)= \begin{cases}H(s-a) & : b>0, s \leq a \\
1 & : b=0\end{cases}
\end{aligned}
$$

Proof. The proof follows immediately from Theorems 3 and 4 .

The p.d.f. and the d.f. of the discrete insurances and annuities considered in DE PRIL (1989) can be derived with the help of Table 2 and Corollary 2.

\section{EXAMPLE}

A person aged $x$ purchases a combination benefit consisting of a $n$-year term life insurance of $I$ payable immediately on his death and a $n$-year deferred whole life annuity of $J$ per annum payable continuously while he survives beyond age $x+n$.

Let the benefit functions of the insurances and annuities defined in Table 1 be denoted by adding a tilde to the usual deterministic symbols. The benefit function of the continuous general insurance defined above is then given by

$$
S=I \bar{\sim}_{x \mathrm{n}\urcorner}^{1}+J_{n \mid \underset{a}{x}}
$$

By using (2.3) and Table 1 this benefit function can be written as a variable of the form (2.4) with $T \equiv T_{x}$ :

$$
S= \begin{cases}I v^{T} & : 0 \leq T<n \\ J \frac{v^{n}-v^{T}}{\delta} & : T \geq n\end{cases}
$$

From Theorem 1 it follows that the p.d.f. of $S$ is given by

$$
f(s)=G_{1}(s)+G_{2}(s)
$$


with

$$
\begin{aligned}
& G_{1}(s)= \begin{cases}r(1) p_{x} \mu_{x+r(1)} /(\delta s) & : I v^{n}<s<I \\
0 & : \text { elsewhere }\end{cases} \\
& G_{2}(s)= \begin{cases}r(2) p_{x} \mu_{x+r(2)} /\left(J v^{n}-\delta s\right) & : 0<s<J v^{n} / \delta \\
0 & : \text { elsewhere }\end{cases}
\end{aligned}
$$

and

$$
\begin{aligned}
& r(1)=-(1 / \delta) \ln (\mathrm{s} / I) \\
& r(2)=-(1 / \delta) \ln \left(v^{n}-(\delta s) / J\right)
\end{aligned}
$$

The d.f. of $S$ follows from Theorem 2:

$$
F(s)=\left({ }_{n} q_{x}-{ }_{\beta} q_{x}\right)_{+}+\left({ }_{a} q_{x}-{ }_{n} q_{x}\right)_{+}
$$

with

$$
\begin{aligned}
& a= \begin{cases}\max \{0, r(2)\} & : s<J v^{n} / \delta \\
\infty & : s \geq J v^{n} / \delta\end{cases} \\
& \beta= \begin{cases}n & : s \leq 0 \\
\max \{0, r(1)\} & : s>0\end{cases}
\end{aligned}
$$

\section{REFERENCES}

Bowers, N. L., Gerber, H. U., Hickman, J.C., Jones, D. A. and Nesbitt, C. J. (1987) Actuarial Mathematics. Society of Actuaries, Itacsa, IL.

DE PrIL, N. (1989) The distributions of Actuarial functions. Bulletin of the Swiss Association of Actuaries, 173-183.

GERBER, H.U. (1986) Lebensversicherungsmathematik. Vereinigung schweizerischer Versicherungsmathematiker, Zürich.

Papoulis, A. (1962) The Fourier Integral and its applications. Mc Graw-Hill, New York.

Pollard, A. H. and Pollard, J. H. (1969) A stochastic approach to actuarial functions. Journal of the Institute of Actuaries, 79-113.

Wolthuis, H. and VAN HoEk, I. (1984) Stochastic models for life contingencies. Insurance: Mathematics and Economics 5, 217-254.

\section{JAN DhaEne}

Instituut voor Actuariële Wetenschappen, K.U. Leuven, Dekenstraat 2, B-3000 Leuven, Belgium. 